\title{
A New Single-Use Bone Marrow Biopsy Needle with Core Retention Design
}

\author{
Anwarul Islam* \\ Department of Medicine, Clinical Associate Professor of Medicine, Division of Hematology/Oncology, Buffalo General Hospital, \\ Buffalo, New York, USA
}

*Corresponding author: Anwarul Islam, Department of Medicine, Clinical Associate Professor of Medicine, Division of Hematology/ Oncology, Buffalo General Hospital, Buffalo, New York, USA

ARTICLE INFO

Received: 慧 June 28, 2019

Published: 慧 July 05, 2019

Citation: Anwarul Islam. A New Single-Use Bone Marrow Biopsy Needle with Core Retention Design. Biomed J Sci \& Tech Res 19(3)-2019. BJSTR. MS.ID.003294.

\begin{abstract}
A needle for obtaining a solid core (trephine) bone marrow biopsy sample from the posterior ilium is described. The needle is comprised of a hollow stainless-steel tube whose front-end diameter is reduced in comparison with the proximal segment. At its terminus the needle is also beveled and has six circumferentially spaced facets thereby forming a sharp cutting edge. The segment of the tube between the longer wider portion of the hollow tube and its smaller reduced diameter front end portion has a series of diamond shaped serrations/flutes on its surface which offer enhanced cutting ability as with a saw to assist in easy penetration of the needle through the dense cortical bone and into the marrow cavity.
\end{abstract}

Keywords: Single-use bone marrow biopsy needle; Core retention bone marrow biopsy needle; Bone marrow biopsy; Needle

\section{Introduction}

Bone marrow (BM) biopsy plays an important role in the investigation, diagnosis and management of various haematological as well as non-haematological malignant conditions [1-4]. Since the development of an improved BM biopsy needle $[5,6]$ and its introduction in a disposable form, there has been a considerable growth in its use. The value of BM biopsy, particularly in different haematological conditions is now well established $[7,8]$. However, loss of core samples during their extraction, i.e., failure to retain the complete biopsy sample within the lumen of the needle as the needle is withdrawn from the patients has been a serious continuing problem. The importance and significance of this particular problem was recognized and was successfully addressed by the development of an improved bone marrow biopsy needle with a core retention device [9]. But until the present time this needle was not available in a disposable form because of technical manufacturing limitations and the cost involved. In recent years technology has emerged which obviate these problems and as a result such a needle can be made economically and in large numbers. Taking advantage of this new technology, a new singleuse bone marrow biopsy needle that retains the original principle of core retention device has now been produced. In addition, a new feature has been added to the design. It consists of a series of surface serrations (flutes) at the tapering transitional portion which ease the boring through the dense cortical bone and entry into the spongy hematopoietic bone. Furthermore, an easy technique of removing the biopsy sample from within the lumen of the needle has also been introduced. In this technique, an insertion aid is used to remove the biopsy specimen. This insertion aid has been designed to receive the distal cutting end of the needle on one side and the front end of the pusher rod on the other side. In this way, the operator can utilize the insertion aid to expel the biopsy sample easily through the proximal end of the needle.

\section{Materials and Methods}

The steel instrument (Figure 1) consists of five parts:

a. The needle (Figure $1 \mathrm{~A}$ ) which has an overall length of 125 $\mathrm{mm}$, a uniform external diameter $3.25 \mathrm{~mm}$, and a constant internal diameter of $2.5 \mathrm{~mm}$ except for the $3.5 \mathrm{~mm}$ distal portion where it is narrowed and has multiple surface serrations/flutes (Figure 2C). The terminus of the needle has six sharp cutting facets (Figure 2B). The internal diameter of the needle's distal portion is less than the overall internal diameter of the needle and begins with a 
short-slanted step of $0.2 \mathrm{~mm}$. This specially designed distal portion (Figure 3E) cuts all the trabecular connections of bone which might keep the biopsy specimen anchored to its base and also holds on to the biopsy sample so that it does not slip out of the needle during the process of its withdrawal. The larger internal diameter of the needle at its proximal segment provides free space (Figure 3B) within the interior of the instrument thus avoiding crushing and compression of the tissue as well as plugging the lumen of the needle.

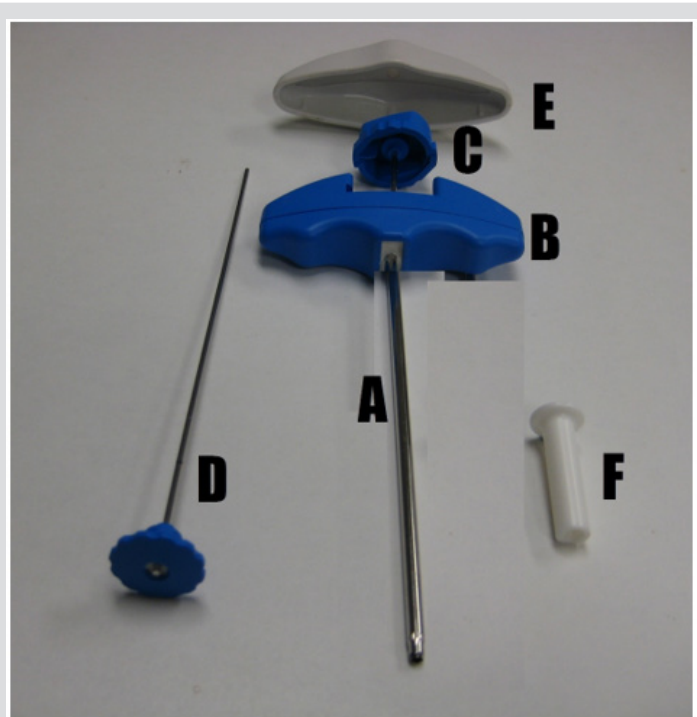

Figure 1: The needle (A), plastic T-bar handle (B), stilette with its plastic head(C), pusher rod (D), plastic cap (E) and the insertion aid $(\mathrm{F})$.

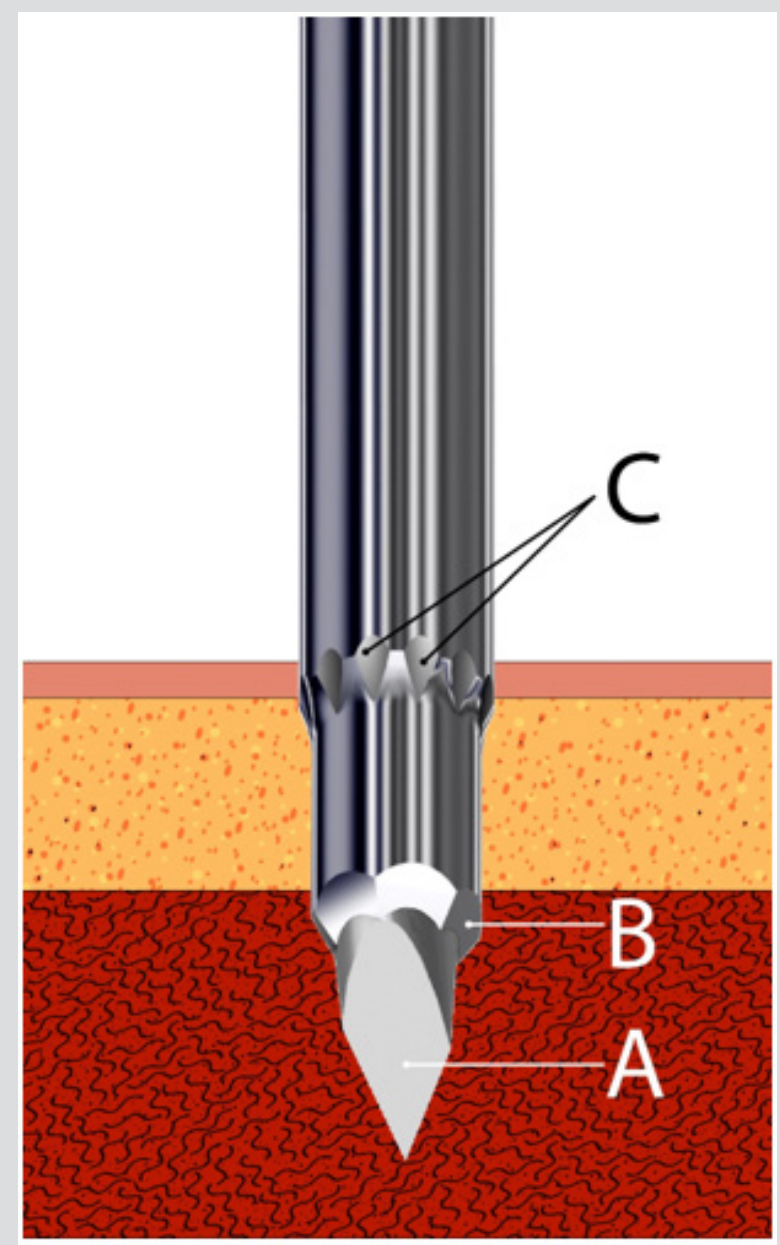

Figure 2: Schematic representation of the distal penetrating and cutting end of the needle showing the elongated trocar pointed stilette (A), sharp cutting facets (B) and the external surface serrations/flutes (C). 


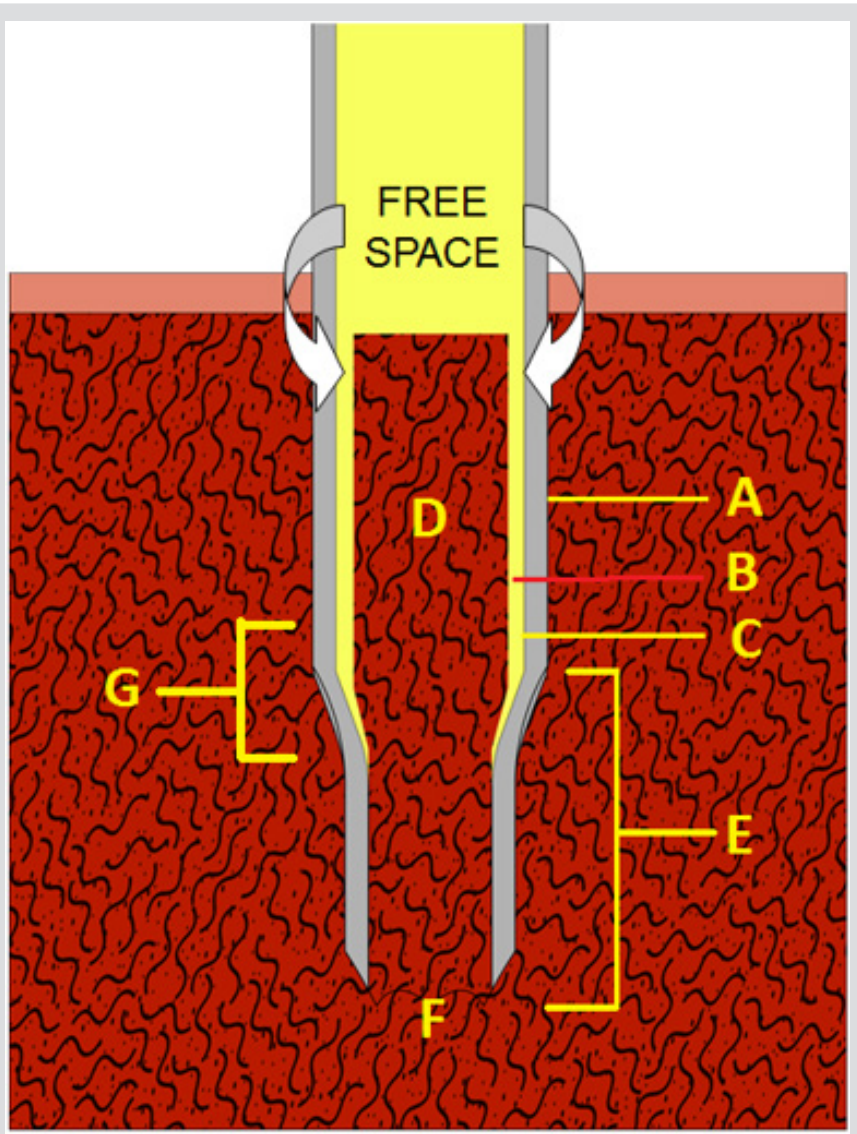

Figure 3: Schematic representation of the biopsy technique. (A) the needle, (B) free space between the core (D) and the internal wall of the needle (C). (E) Specially tooled distal cutting end of the needle which cuts the trabecular connections of the core at its base $(F)$ and holds the core sample during the process of its extraction so that it does not slip out of the needle. (G) shows the area of the distal cutting end of the needle where it joins the main body of the needle which is wider.

It also facilitates easy delivery of the biopsy sample through the proximal end of the needle. The introduction of the needle through the cortical bone could potentially be hampered by the wider proximal portion of the needle (Figure 3G). To prevent this obstruction and assist an easy, smooth entry of the needle into the marrow cavity, multiple surface serrations/flutes (Figure 2C) are provided at the junction of the distal narrower portion with its adjoining wider proximal portion. These surface serrations (flutes) provide a means of drilling/cutting as the needle is being advanced through the hard-cortical bone while rotating the needle by clockwise and counter-clockwise rotary motions. The proximal end of the needle is fitted with a large plastic T- bar handle (Figure 1B) for a firm grip.

b. The stilette (Figure 1C) is a solid steel shaft of $2.0 \mathrm{~mm}$ in diameter. It ends with a $3.0 \mathrm{~mm}$ long three- faceted, sharply pointed tip (Figure 2A) which projects beyond the cutting edge of the needle in order to protect the sharp cutting facets of the needle. The stilette also provides a means of easy penetration of the cortical bone. The proximal end of the stilette is fitted with a round knurled plastic knob (head) (Figure 1C) which fits into the proximal (top) end of the needle.

c. The plastic cap (Figure 1E) fits and covers the top of the plastic T-bar handle (Figure 1B). It also fits snugly in the palm of the operator's hand and is used to avoid discomfort during the biopsy procedure.

d. The insertion aid (Figure $1 \mathrm{~F}$ ) is a tubular mass of plastic $30 \mathrm{~mm}$ long and $5 \mathrm{~mm}$ wide except at the end that fits the mouth of the needle where it is flat and $10 \mathrm{~mm}$ in diameter. It is centrally hollowed out to receive and passage of the pusher during removal of the biopsy specimen through the proximal end of the needle.

e. The pusher (Figure 1D) is a solid steel rod of $2.0 \mathrm{~mm}$ in diameter attached to a knurled, flat plastic disk. The Pusher rod is used to remove the biopsy specimen from within the lumen of the biopsy needle with the help of the insertion aid which guides the pusher rod into the mouth needle.

\section{Biopsy Procedure (Figure 4)}

The instrument has been designed to obtain bone marrow biopsy specimens from the right or left posterior ilium. The patient is placed in a right or left lateral decubitus position with the top knee bent forward and drawn up and back comfortably flexed, or in the prone position with a pillow beneath the hips. The site of posterior iliac crest is first identified by palpation and the overlying skin is prepared with an antiseptic and draped. Then the skin, subcutaneous tissue, and the periosteum are infiltrated with a local anesthetic ( 1 or $2 \%$ lidocaine). A small $3 \mathrm{~mm}$ skin incision is 
made with a sharp pointed scalpel blade. The biopsy needle with the stilette and plastic cap in place is advanced slowly through the incision, pointing towards the anterior superior iliac spine (Figure 5) and when the posterior iliac crest is reached it is then penetrated by gentle rotary motions of the needle. Once the cortex is penetrated the needle gets locked in the bone. The stilette is then removed by twisting the knob of the stilette anti-clockwise with one hand while holding the needle in place with the other (Figure 6).

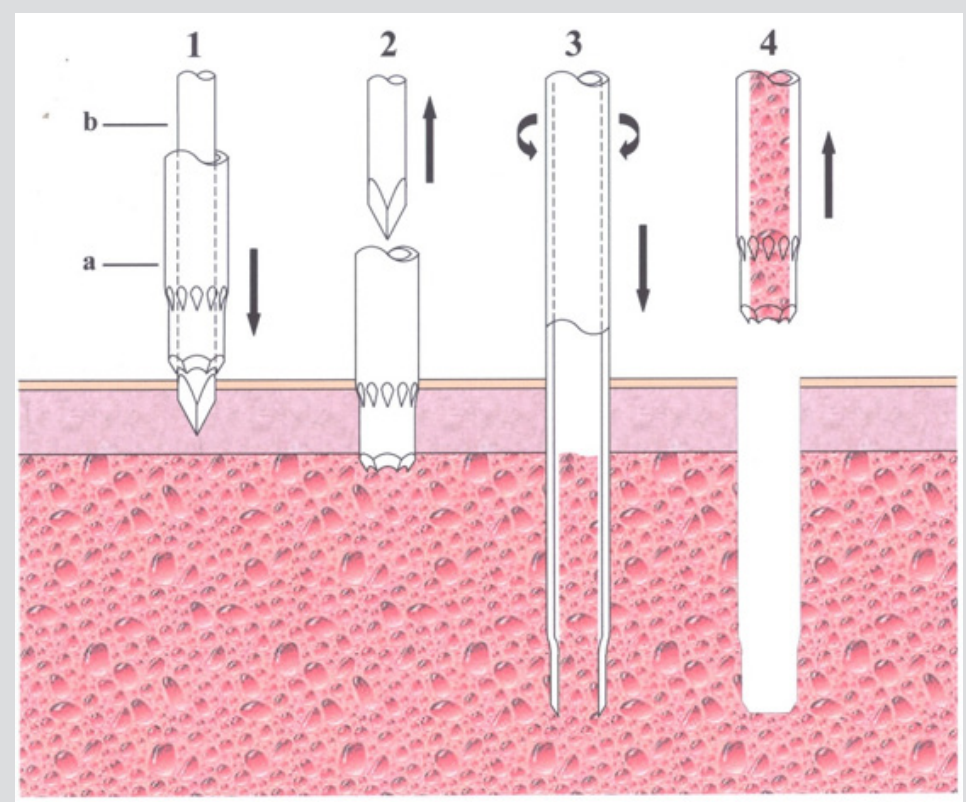

Figure 4: Schematic representation of the biopsy procedure. The needle (a) with the stilette (b) in place is inserted to the bone (1). The cortex of the posterior ilium is then penetrated by gentle rotary motion of the needle. Once this penetration has been achieved, the stilette is withdrawn (2). The needle is then advanced with slow, steady and controlled clockwisecounterclockwise rotary motion (3). When an adequate depth is reached the needle is rotated several times along its long axis and then withdrawn with a straight pull (4). No rocking, sculling movement or change in the direction of the tip of the needle are necessary.

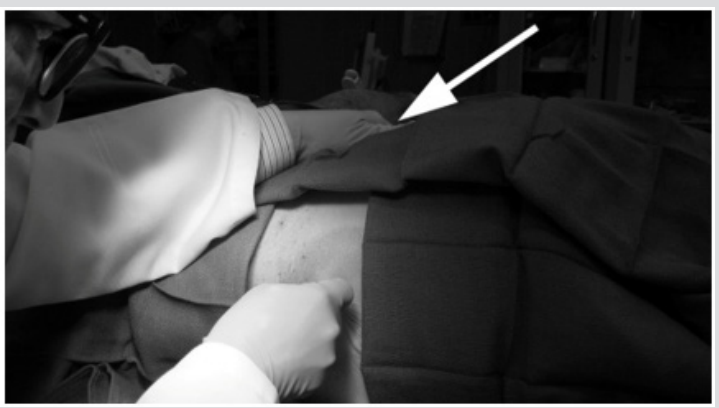

Figure 5: Demonstrates the introduction of the needle. As stated in the text it is useful to put one hand over the anterior iliac crest with the middle or index finger on the anterior superior iliac spine as shown in the photograph (arrow). This approach not only helps stabilize the patient's pelvis but also helps in the guidance of the needle towards the anterior superior iliac spine.

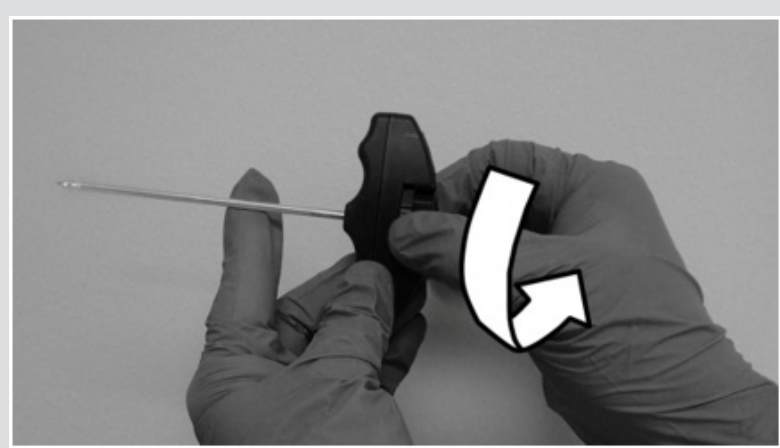

Figure 6: Demonstrates the unlocking of the stilettet from the T-bar handle by anti-clockwise twisting the knurled head/knob of the trocar (arrow) and then withdrawing the stilette with a straight pull. 
The biopsy needle is then advanced with slow, steady and controlled clockwise-counterclockwise rotary motions until an adequate depth is reached (about $20 \mathrm{~mm}$ ). The biopsy needle is then rotated completely several times about its long axis to sever all the trabecular connections at its base and sever the core sample from the surrounding spongy bone. It is then slowly withdrawn with a straight pull and gentle alternating rotary motions. No rocking, sculling or gyratory movements or change in the direction of the tip of the needle are necessary. Once the needle is completely withdrawn from the patient, the insertion aid is attached to the distal cutting end (mouth) of the needle (Figure 7). The biopsy specimen is then pushed out of the needle with the pusher rod which is introduced through the opposite end of the insertion aid (Figure 8). Once the biopsy procedure is complete a gauze dressing is applied, and an adhesive tape is placed to keep the gauge dressing in place. The patient is then instructed to lie flat on his/her back for 10-15 minutes or longer if the patient has a low platelet count, on aspirin or on anticoagulants.

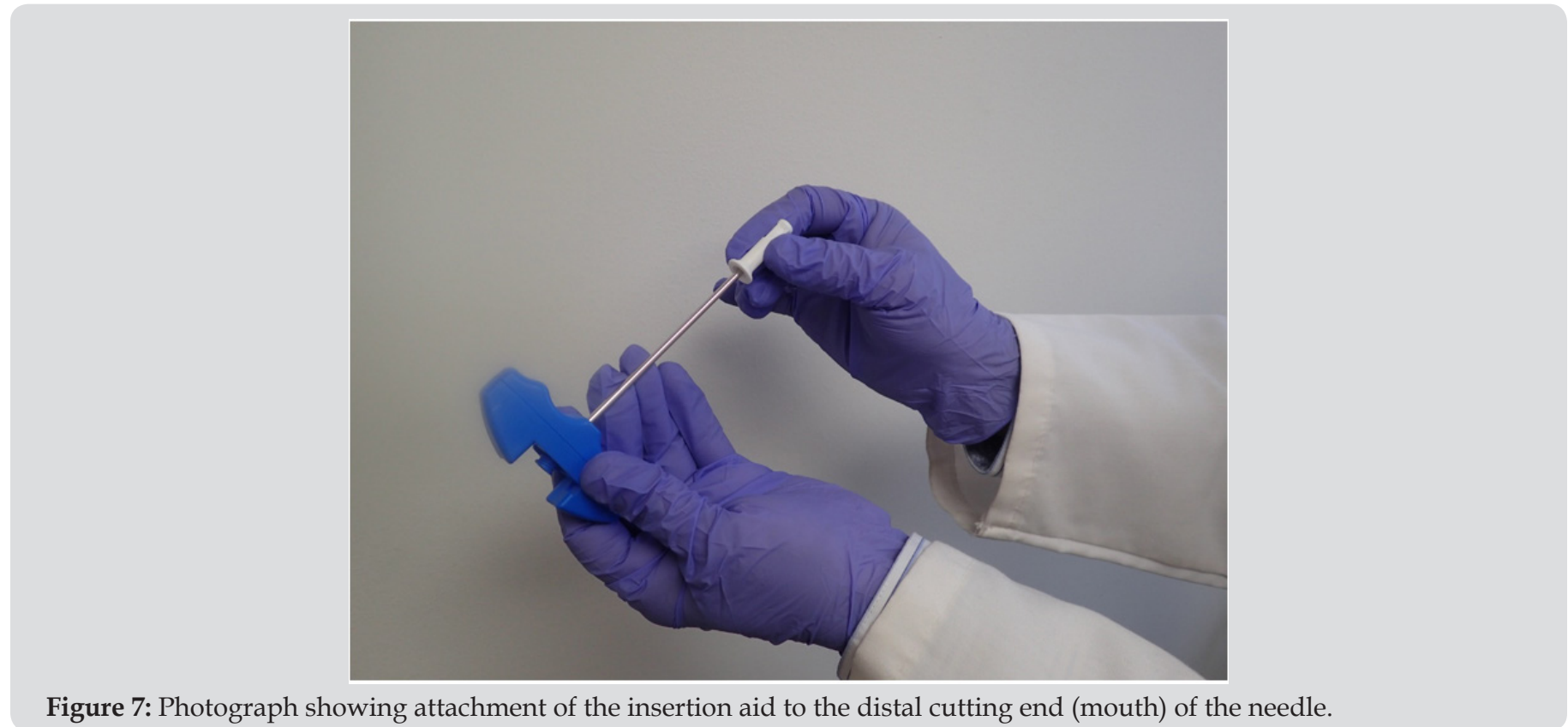

Figure 7: Photograph showing attachment of the insertion aid to the distal cutting end (mouth) of the needle.

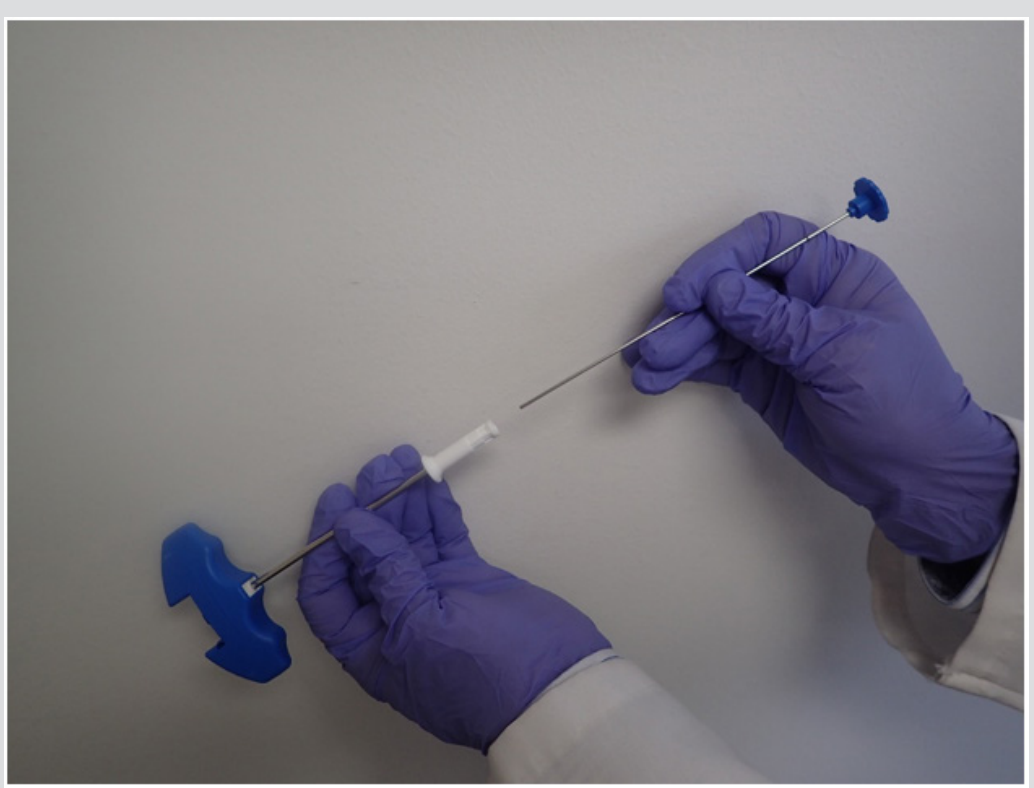

Figure 8: Photograph showing introduction of the stilette through the insertion aid to push the core sample out of the needle.

\section{Results}

The needle has been extensively tested. An adequate sample was obtained with each attempt. Bony trabeculae were clean cut and crushing artefact was almost negligible even at the edges of the biopsy specimen. The quality of the specimen obtained with this needle has been excellent in each case (Figure 9). 


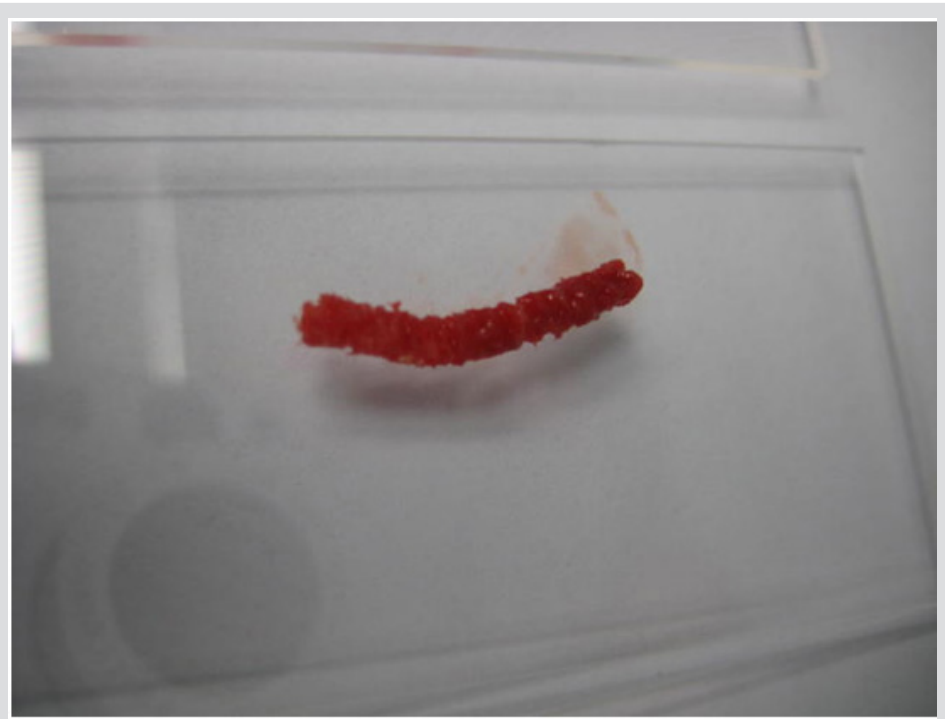

Figure 9: A core sample of bone marrow obtained with this needle. Note the long and uniform core of marrow tissue obtained by this needle.

\section{Discussion}

BM biopsy, since its introduction by Ghedini [10] has achieved significant importance in the field of medicine, and hematology and oncology in particular. It is now routinely used in the investigations, diagnosis and management of various haematological as well as non-haematological malignant conditions. The Jamshidi needle, which popularized the technique of bone marrow core biopsy in the 60 's, had one major problem: its inability to consistently retain the biopsy specimens within the lumen of the needle at each and every attempt of usage. The Jamshidi needle had this problem because it did not have a core retention feature. Because of this the operator had to resort to some extraneous movements with the needle such as rocking, sculling or gyratory movements or change in the direction of the tip of the needle to capture and secure a solid core sample.

Although the 'sample or core losing' problem of the Jamshidi needle was soon recognized and was addressed by the development of a new bone marrow biopsy needle with a core retention design [9], this particular needle has not been available in a disposable - i.e., single-use form until the present time. To overcome the problem of losing the core biopsy specimen several different single use needles have been Introduced [11- 13]. Each of these needles features different methods of securing a core sample during withdrawal of the needle from the patient. Unfortunately, all of these alternatives significantly complicate the procedure by introducing multiple steps and an assortment of supplementary parts and components. Indeed, in some cases, these needles contain six or more additional parts. Furthermore, these core-capturing devices (e.g., sleeve, coil, spoon, marrow acquisition cradle) may cause a crush artefact at the edges of the biopsy specimen and reduce the amount of marrow tissue available for histological evaluation.

Accordingly, the advantage of this new needle lies not only in its simplicity but also a reduced number of parts and steps required to complete the biopsy procedure. This new needle essentially has only four parts - the needle, the stilette, the pusher and an insertion aid. In addition, the distal cutting end of the needle, which bears the biopsy retention device, has also been fitted with multiple surface serrations or flutes on the tapering transition portion, between the main wider portion of the hollow needle and its reduced-diameter front end portion (Figure 2C). These diamond shaped surface serrations (flutes) acts like a saw when rotated and assist penetration of the bone by sawing through the cortical bone and facilitate the entry of the main wider portion of the hollow needle into the marrow cavity. The internal diameter of the distal narrower portion is less than the overall internal diameter of the proximal portion. This specially designed distal $(3.5 \mathrm{~mm})$ portion of the needle with sharp cutting facets not only cuts all the trabecular connections at its base which might keep the biopsy specimen firmly anchored to its base but also holds the core sample with pincer-like action so that it does not slip out of the needle during its extraction (Figure 3E).

Once the biopsy specimen has entered the needle and has passed beyond the narrower segment the sample is able to expand to some extent. Here, the internal step acts as a shoulder which prevents the slightly expanded biopsy sample from slipping out of the needle during its withdrawal. In addition, the larger internal diameter of the needle provides free space (Figure 3C) within the interior lumen of the needle. This prevents crushing and compression of the tissue as well as plugging the lumen of the needle. This also allows easy delivery of the biopsy specimen through the proximal end of the needle. The distal cutting end of the needle - where it joins the main body of the needle - is wider (Figure 3G). As a result, the introduction of the needle through the hard-cortical bone may be hampered by the wider proximal portion of the needle. To avoid this potential obstruction and promote an easy and smooth entry of the needle through the cortical bone multiple surface serrations are provided at this junction. Furthermore, the biopsy technique 
with this needle is simple and does not require any manipulation of the biopsy needle within the patient in order to secure/capture the biopsy specimen. This in turn makes this potentially uncomfortable procedure more tolerable to the patient.

\section{References}

1. McFarland W, Dameshek W (1958) Biopsy of bone marrow with the Vim-Silverman needle. JAMA 166(12): 1464-1466.

2. Ellman L (1976) Bone marrow biopsy in the evaluation of lymphoma, carcinoma and granulomatous disorders. Am J Med 60(1): 1-7.

3. Burke JS (1978) The value of the bone marrow biopsy in the diagnosis of hairy cell leukaemia. Am J Clin Pathol 70(6): 876-884.

4. Brunning RD, Bloomfield CD, McKenna RW, Peterson L (1975) Bilateral trephine bone marrow biopsies in lymphoma and other neoplastic disease. Ann Int Med 82(3): 365-366.

5. Jamshidi K, Swaim WR (1971) Bone marrow biopsy with unaltered architecture: a new biopsy device. J lab Clin Med 77(2): 335-342.

ISSN: 2574-1241

DOI: 10.26717/BJSTR.2019.19.003294

Anwarul Islam. Biomed J Sci \& Tech Res

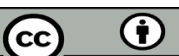

This work is licensed under Creative Commons Attribution 4.0 License

Submission Link: https://biomedres.us/submit-manuscript.php
6. Jamshidi K, Windschitl HE, Swaim WR (1971) A new biopsy needle for bone marrow. Scand J Haematol 8(1): 69-71.

7. Brynes RK, McKenna RW, Sundberg RD (1978) Bone marrow aspiration and trephine biopsy an approach to a thorough study. Am J Clin pathol 70(5): 753-759.

8. Hyun BH, Gulati GL, Ashton JK (1988) Bone marrow examination: techniques and interpretation. Hematol Oncol Clin N Am 2(4): 51-523.

9. Islam A (1982) A new bone marrow biopsy needle with core securing device. J Clin Pathol 35(3): 359-364.

10. Ghedini G (1908) Studi sulla patologia del midello osseo umano vivente. I. Punctura explorative tecnica. Clin Med Ital 47: 724-727.

11. Goldenberg AS, Tiesinga JJ (2001) Clinical experience with a new specimen capturing bone marrow biopsy needle. Am J Hematol 68(3): 189-193.

12. Jamshidi Crown ${ }^{\mathrm{TM}}$ bone marrow biopsy needle and marrow acquisition cradle by Care Fusion/BD.

13. Core Lock ${ }^{\mathrm{TM}}$ bone marrow biopsy system by Worldwide Medical technologies/Argon.

$\begin{array}{ll}\text { BIOMEDICAL } & \text { Assets of Publishing with us } \\ \text { RESEARCHES } & \text { - Global archiving of articles } \\ & \text { - Immediate, unrestricted online access } \\ & \text { - Rigorous Peer Review Process } \\ \end{array}$

